Теорія Ймовір. та Матем. Статист. Вип. 81, 2010
Theor. Probability and Math. Statist.

No. 81, 2010, Pages 131-146

S 0094-9000(2011)00815-0

Article electronically published on January 20, 2011

\title{
FUNCTIONAL LIMIT THEOREMS FOR STOCHASTIC INTEGRALS WITH APPLICATIONS TO RISK PROCESSES AND TO SELF-FINANCING STRATEGIES IN A MULTIDIMENSIONAL MARKET. I
}

UDC 519.21

\author{
YU. S. MISHURA, G. M. SHEVCHENKO, AND YU. V. YUKHNOVS'KII
}

\begin{abstract}
We study sufficient conditions for the weak convergence of stochastic integrals with respect to processes of bounded variation, martingales, or semimartingales. A semimartingale theorem is extended to the multidimensional case. We apply a limit procedure and pass from processes of bounded variation to risk processes. An "inverse" problem for the weak convergence is also considered.
\end{abstract}

\section{INTRODUCTION}

In the first part of the paper, we deal with the conditions for the weak convergence of stochastic integrals

$$
\int_{0}^{b} \xi_{n}(t) d X_{n}(t)
$$

with respect to processes $\left\{X_{n}, n \geq 1\right\}$ of bounded variation, martingales, or semimartingales. We consider the convergence of the corresponding probability measures in the Skorokhod space $D[0, b], b>0$. Many papers are devoted to finding conditions for the weak convergence of stochastic integrals; an extensive bibliography and the most general conditions can be found in the book [5, Chapter IX].

There is a number of differences between conditions introduced in $[5$ and those considered in this paper. First, a different convergence of the sequence of stochastic processes $\xi_{n}(t)$ is considered in this paper; namely, we study the weak convergence of finitedimensional distributions of the processes. Further, most of the conditions in [5] imposed on the sequence of semimartingales $X_{n}$ are related to the triplet of predictable characteristics $(B, C, \nu)$. Checking these conditions leads to several complications, since it requires finding these characteristics.

In this paper we are not aimed at obtaining a canonical decomposition or the triplet of its predictable characteristics. The assumptions imposed on the sequence of semimartingales $X_{n}$ are expressed in terms of components of an arbitrary decomposition involving a square integrable martingale $M_{n}$ and a process of bounded variation $B_{n}$.

Conditions for the weak convergence of stochastic integrals with respect to processes of bounded variation are given in Section 2. We provide an example of the application of

2010 Mathematics Subject Classification. Primary 60G44, 60F05, 60B12.

Key words and phrases. Stochastic integrals, functional limit theorems, weak convergence, semimartingales.

The first two authors are grateful to the European Commissions for support in the framework of the program "Marie Curie Actions", grant PIRSES-GA-2008-230804. 
the main result of this section to risk processes. Conditions for the convergence of stochastic integrals with respect to martingales are presented in Section 3. The convergence of stochastic integrals with respect to martingales is also considered in the paper [2] We describe the differences between these two results. The main result on the weak convergence of stochastic integrals with respect to semimartingales in the Skorokhod space is given in Section 4. We do not provide the most general conditions here; rather, we exhibit conditions that are easy to check. An application of the theorem of Section 5 to self-financing strategies will be given in the second part of this paper.

\section{Convergence of integrals over Stochastic PROCESSES OF BOUNDED VARIATION}

Let

$$
\left(\Omega^{n}, \mathcal{F}^{n},\left(\mathcal{F}_{t}^{n}\right)_{t \in \mathbf{R}_{+}}, \mathrm{P}^{n}\right)
$$

be a stochastic basis for every $n$, and let $\left\{\mu_{n}(t), n \in \mathbf{Z}_{+}, t \in \mathbf{R}_{+}\right\}$be a sequence of processes whose trajectories almost surely have bounded variation on every bounded interval $[0, b]$, do not have discontinuities of the second kind, and are right continuous. Also let $\left\{\xi_{n}(t), n \in \mathbf{Z}_{+}, t \in \mathbf{R}_{+}\right\}$be a sequence of processes whose trajectories do not have discontinuities of the second kind and are right continuous. Fix a countable and everywhere dense set $T$ in $\mathbf{R}_{+}$. Put $T_{b}:=T \cap[0, b)$.

Denote by $L_{T_{b}}$ the class of all sequences

$$
\alpha_{k}=\left\{0=t_{0 k}<t_{1 k}<\cdots<t_{k_{b} k}<b\right\}
$$

of finite partitions of the interval $[0, b)$ such that

1) $\alpha_{k} \subseteq \alpha_{k+1} \subseteq T_{b}$

2) for every $t \in T_{b}$, there exists $k(t)$ such that $t \in \alpha_{k}$ for $k>k(t)$.

We say that condition (A) holds if the limit

$$
S\left(\xi_{n}, \mu_{n}, 0, b\right):=\lim _{k \rightarrow \infty} \sum_{i=1}^{k_{b}+1} \xi_{n}\left(t_{i-1 k}\right)\left(\mu_{n}\left(t_{i k}\right)-\mu_{n}\left(t_{i-1 k}\right)\right)
$$

exists with probability one for all $b>0, n \in \mathbf{Z}_{+}$, and for all sequences $\alpha_{k} \in L_{T_{b}}$, $\alpha_{k}=\left\{0=t_{0 k}<t_{1 k}<\cdots<t_{k_{b} k}<b\right\}$ such that $t_{k_{b} k}<b \leq t_{k_{b}+1 k}$.

If condition (A) holds, then the random variable $S\left(\xi_{n}, \mu_{n}, 0, b\right)$ is equal to the Riemann-Stieltjes integral $\int_{0}^{b} \xi_{n}(t) d \mu_{n}(t)$ if this integral exists (see [8]).

Put

$$
\begin{gathered}
\triangle_{i k} x:=x\left(t_{i k}\right)-x\left(t_{i-1 k}\right), \quad \omega_{i k} x=\sup _{t_{i-1} \leq s<t \leq t_{i k}}|x(t)-x(s)|, \\
k_{t}=\sup \left\{i: t_{i k} \leq t\right\} .
\end{gathered}
$$

In what follows, the symbol " $\Rightarrow$ " stands for the weak convergence of finite-dimensional distributions. We also recall the notion of convergence of probability measures in the Skorokhod space.

Denote by $D[0, b]$ the space of functions on the interval $[0, b]$ that have no discontinuities of the second kind and are right continuous. We equip this space with the following metric $d_{0}(\cdot, \cdot)$ :

$$
d_{0}(x, y)=\inf _{\lambda \in \Lambda}\left\{\sup _{0 \leq t \leq b}|x(t)-y(\lambda(t))|+\sup _{0 \leq s<t \leq b}\left|\log \frac{\lambda(t)-\lambda(s)}{t-s}\right|\right\}
$$

for $x, y \in D[0, b]$, where $\Lambda$ is the set of strictly increasing continuous mappings of the interval $[0, b]$ to itself. It is well known that the metric $d_{0}(\cdot, \cdot)$ is equivalent to the 
Skorokhod metric

$$
d(x, y)=\inf _{\lambda \in \Lambda}\left\{\sup _{0 \leq t \leq b}|x(t)-y(\lambda(t))|+\sup _{0 \leq t \leq b}|\lambda(t)-t|\right\},
$$

which generates the Skorokhod topology (see, for example, [3], Chapter 3).

Consider a family of probability measures $\left(Q^{n}\right)_{n \geq 1}$ defined on the space $(D[0, b], \mathcal{D})$, where $\mathcal{D}$ is the $\sigma$-algebra of Borel sets generated by the metric $d_{0}(\cdot, \cdot)$. We say that a sequence of measures $Q^{n}$ weakly converges to a probability measure $Q$ on $(D[0, b], \mathcal{D})$ if

$$
\lim _{n \rightarrow \infty} \int_{[0, b]} f(x) d Q^{n}=\int_{[0, b]} f(x) d Q
$$

for all real functions $f=f(x), x \in[0, b]$, bounded and continuous in the topology generated by the Skorokhod metric.

The symbol " $\stackrel{D[0, b]}{\rightarrow}$ " stands for the above convergence of probability measures in the Skorokhod topology on the interval $[0, b]$.

We introduce the following notation:

$$
\triangle_{D}(x(\cdot), \delta, b):=\sup _{0 \leq t<t^{\prime}<t^{\prime \prime}<t+\delta \leq b}\left(\left|x\left(t^{\prime \prime}\right)-x\left(t^{\prime}\right)\right| \wedge\left|x\left(t^{\prime}\right)-x(t)\right|\right)
$$

and $\mathcal{T}_{b}\left(\mathcal{F}^{n}\right)=\left\{0 \leq \tau \leq b, \tau\right.$ is an $\mathcal{F}^{n}{ }^{n}$-stopping time $\}$.

Theorem 1. 1. Let condition (A) hold. Assume that

$\left(\mathrm{A}_{1}\right)$ 1) $\left(\xi_{n}(t), \mu_{n}(t)\right), t \in T_{b} \Rightarrow\left(\xi_{0}(t), \mu_{0}(t)\right), t \in T_{b}$, for all $b>0$ as $n \rightarrow \infty$;

2) for all $b>0$ and $\alpha>0$,

$$
\lim _{k \rightarrow \infty} \limsup _{n \rightarrow \infty} \mathrm{P}^{n}\left\{\sum_{i=1}^{k_{b}+1} \omega_{i k} \xi_{n} \omega_{i k} \mu_{n}>\alpha\right\}=0 .
$$

Then

$$
S\left(\xi_{n}, \mu_{n}, 0, b\right) \Rightarrow S\left(\xi_{0}, \mu_{0}, 0, b\right) \quad \text { for all } b>0 .
$$

2. Assume additionally that

$\left(\mathrm{A}_{2}\right)$ 1) for all $b>0$,

$$
\lim _{C \rightarrow \infty} \limsup _{n \rightarrow \infty} \mathrm{P}^{n}\left\{\sup _{0 \leq t \leq b}\left|\xi_{n}(t)\right| \geq C\right\}=0 ;
$$

2) for all $\alpha>0$ and $b>0$,

$$
\lim _{\delta \rightarrow 0} \limsup _{n \rightarrow \infty} \mathrm{P}^{n}\left\{\triangle_{D}\left(\left|\mu_{n}\right|(\cdot), \delta, b\right)>\alpha\right\}=0
$$

or

$\left(\mathrm{A}_{3}\right)$ 1) $\xi_{n}(t)$ and $\mu_{n}(t)$ are $\mathcal{F}_{t}^{n}$-measurable, $t \in \mathbf{R}_{+}$;

2) for all $\alpha>0$ and $b>0$,

$$
\lim _{\delta \rightarrow 0} \limsup _{n \rightarrow \infty} \sup _{\tau \in \mathcal{T}_{b}\left(\mathcal{F}^{n}\right)} \mathrm{P}^{n}\left\{\sup _{0 \leq t \leq \delta} \sum_{i=k_{\tau}}^{k_{\tau+t}+1}\left|\xi_{n}\left(t_{i-1 k}\right)\right|\left|\Delta_{i k} \mu_{n}\right|>\alpha\right\}=0 .
$$

Then

$$
S\left(\xi_{n}, \mu_{n}, 0, \cdot\right) \stackrel{D[0, b]}{\rightarrow} S\left(\xi_{0}, \mu_{0}, 0, \cdot\right), \quad n \rightarrow \infty,
$$

for all $b>0$. 
Proof. 1. Consider the following sequence of random variables:

$$
S_{T, n, k}=\sum_{i=1}^{k_{b}+1} \xi_{n}\left(t_{i k}\right) \Delta_{i k} \mu_{n} .
$$

If condition (A) holds, then

$$
S_{T, 0, k} \rightarrow \int_{0}^{b} \xi_{0} d \mu_{0} \quad \text { a.s. }
$$

On the other hand, condition $\left(\mathrm{A}_{1}\right)$ implies that

$$
S_{T, n, k} \Rightarrow S_{T, 0, k}
$$

as $n \rightarrow \infty$ for all $k$.

Put

$$
\begin{aligned}
& S^{+}\left(\alpha_{k}, \xi_{n}, \mu_{n}\right)=\sum_{i=1}^{k_{b}+1} \sup _{t_{i-1} \leq t<t_{i k}} \xi_{n}(t)\left(\mu_{n}\left(t_{i k}\right)-\mu_{n}\left(t_{i-1}\right)\right), \\
& S^{-}\left(\alpha_{k}, \xi_{n}, \mu_{n}\right)=\sum_{i=1}^{k_{b}+1} \inf _{t_{i-1} \leq t<t_{i k}} \xi_{n}(t)\left(\mu_{n}\left(t_{i k}\right)-\mu_{n}\left(t_{i-1 k}\right)\right) .
\end{aligned}
$$

By definition, $-\infty<S^{-}\left(\alpha_{k}, \xi_{n}, \mu_{n}\right) \leq S^{+}\left(\alpha_{k}, \xi_{n}, \mu_{n}\right)<\infty$ P-almost surely, and the sums $S^{+}\left(\alpha_{k}, \xi_{n}, \mu_{n}\right)\left(S^{-}\left(\alpha_{k}, \xi_{n}, \mu_{n}\right)\right)$ do not increase (do not decrease) with respect to $k$ for any sequence of partitions $\alpha_{k} \in L_{T_{b}}$. Thus the limits

$$
S_{T}^{ \pm}\left(\xi_{n}, \mu_{n}\right)=\lim _{k \rightarrow \infty} S^{ \pm}\left(\alpha_{k}, \xi_{n}, \mu_{n}\right)
$$

exist and are finite almost surely. It is obvious that

$$
\begin{aligned}
& S^{+}\left(\alpha_{k}, \xi_{n}, \mu_{n}\right) \geq S_{T, n, k}, \\
& S^{-}\left(\alpha_{k}, \xi_{n}, \mu_{n}\right) \leq S_{T, n, k} .
\end{aligned}
$$

Using condition $\left(\mathrm{A}_{1}\right) 2$ ), we obtain from (2) and (3) that

$$
\begin{aligned}
\lim _{k \rightarrow \infty} & \varlimsup_{n \rightarrow \infty} \mathrm{P}^{n}\left\{\left|S_{T}^{ \pm}\left(\xi_{n}, \mu_{n}\right)-S_{T, n, k}\right|>\alpha\right\} \\
& \leq \lim _{k \rightarrow \infty} \varlimsup_{n \rightarrow \infty} \mathrm{P}^{n}\left\{S^{+}\left(\alpha_{k}, \xi_{n}, \mu_{n}\right)-S^{-}\left(\alpha_{k}, \xi_{n}, \mu_{n}\right)>\alpha\right\} \\
& =\lim _{k \rightarrow \infty} \lim _{n \rightarrow \infty} \mathrm{P}^{n}\left\{\sum_{i=1}^{k_{b}+1} \omega_{i k} \xi_{n} \omega_{i k} \mu_{n}>\alpha\right\}=0 .
\end{aligned}
$$

Now the first part of the theorem follows from (2)-(5) (see Theorem 4.2 in [3]).

2. Assume that condition $\left(\mathrm{A}_{2}\right)$ holds. The proof then follows from the easy estimate

$$
\triangle_{D}\left(S\left(\xi_{n}, \mu_{n}, 0, \cdot\right), \delta, b\right) \leq \sup _{0 \leq t \leq b}\left|\xi_{n}(t)\right| \cdot \triangle_{D}\left(\left|\mu_{n}\right|(\cdot), \delta, b\right)
$$

and from the weak convergence of the finite-dimensional distributions of $S\left(\xi_{n}, \mu_{n}, 0, \cdot\right)$.

Now assume that condition $\left(\mathrm{A}_{3}\right)$ holds. Then Theorem 1 of [1], Part 2, Chapter $6, \S 3$, implies that the sequence $S\left(\xi_{n}, \mu_{n}, 0, \cdot\right)$ is relatively compact in the Skorokhod topology. The weak convergence of the finite-dimensional distributions of the sequence of stochastic integrals implies their convergence in the Skorokhod topology.

Remark 1. Note that $\mu_{0}$ in Theorem 1 is an arbitrary process (even a process of unbounded variation) such that the limit $S\left(\xi_{0}, \mu_{0}, 0, b\right)$ on the right hand side of (1) exists almost surely for all $b>0$.

Here is an example of applications of Theorem 1 . 
Example 1. Let $U_{n}(t), n \geq 0$, be the gain process of an insurance company, defined on a probability space $(\Omega, \mathcal{F}, \mathrm{P})$, and let

$$
U_{n}(t, \omega):=\pi_{n}(t)-\sum_{k=1}^{N_{n}(t, \omega)} X_{k}^{(n)}(\omega),
$$

where $\pi_{n}$ is a sequence of processes with continuous and nondecreasing trajectories (one can treat $\pi_{n}$ as the premium income processes); $\left\{X_{k}^{(n)}, k \geq 1\right\}$, for any $n>0$, is a sequence of independent identically distributed random variables with distribution functions $F_{n} ; N_{n}(t, \omega)$ is a sequence of point processes whose trajectories do not have discontinuities of the second kind and are right continuous, $N_{n}(0, \omega)=0$. One can treat the sums $\sum_{k=1}^{N_{n}(t, \omega)} X_{k}^{(n)}(\omega)$ as a sequence of insurance premiums. Let

$$
0<T_{1}^{n}(\omega)<T_{2}^{n}(\omega)<\cdots
$$

be a sequence of jumps of $N_{n}, \triangle N_{n}\left(T_{k}^{n}\right)=1$. The reserve capital is defined via the accumulator $\varphi_{n}$ as follows:

$$
\begin{aligned}
R_{n}(t) & :=\varphi_{n}(t) u+\int_{0}^{t} \varphi_{n}(t-s) d U_{n}(s) \\
& =\varphi_{n}(t) u+\int_{0}^{t} \varphi_{n}(t-s) d \pi_{n}(s)-\sum_{k=1}^{N_{n}(t)} \varphi_{n}\left(t-T_{k}^{n}\right) X_{k}^{n}(\omega)
\end{aligned}
$$

(see [4]), where $u>0$ is the initial reserve capital and where $\varphi_{n}: \mathbf{R}_{+} \rightarrow \mathbf{R}_{+}$is a nonrandom continuous nondecreasing function (as a rule, $\varphi_{n}(t)=\exp \{\delta t\}$ for some $\delta>0)$. The following result is a straightforward corollary of Theorem 1 and Theorem 4 in 4 .

Fix some $b>0$ and put

$$
X_{n}(t):=B_{n}^{-1}\left(\sum_{k=1}^{\left[\alpha_{n} t\right]} X_{k}^{(n)}-A_{n}(t)\right),
$$

where $\alpha_{n} \uparrow \infty$ and $B_{n} \uparrow \infty$ as $n \rightarrow \infty$ and where $A_{n}(t)$ is a nonrandom nondecreasing function.

Theorem 2. Assume that

1) $\varphi_{n}(t) \rightarrow \varphi_{0}(t)$ pointwise as $n \rightarrow \infty$;

2) $\left(\pi_{n}(t), X_{n}(t), N_{n}(t) / \alpha_{n}, t \in T_{b}\right) \Rightarrow\left(\pi_{0}, X_{0}(t), N_{0}(t), t \in T_{b}\right)$;

3) $S\left(\varphi_{0}(b-\cdot), X_{0}(\cdot), 0, b\right)$ is well defined;

4) for all $\alpha>0$,

$$
\lim _{k \rightarrow \infty} \limsup _{n \rightarrow \infty} \mathrm{P}\left\{\sum_{i=1}^{N_{n}(b)} \Delta_{l_{i}^{n} k} \varphi_{n}(b-\cdot)\left|X_{i}^{n}\right|>\alpha\right\}=0,
$$

where $l_{i}^{n}$ is such that $t_{l_{i}^{n}-1 k} \leq T_{i}^{n}<t_{l_{i}^{n} k}$;

5) for all $\alpha>0$,

$$
\lim _{k \rightarrow \infty} \limsup _{n \rightarrow \infty} \mathrm{P}\left\{\sum_{i=1}^{k} \triangle_{i k} \varphi_{n}(b-\cdot) \triangle_{i k} \pi_{n}>\alpha\right\}=0 .
$$

Then the reserve capital processes weakly converge,

$$
R_{n}(b) \Rightarrow \varphi_{0}(b) u+\int_{0}^{b} \varphi_{0}(b-s) d \pi_{0}(s)-\int_{0}^{b} \varphi_{0}(b-s) d Y_{0}(s),
$$

and the limit process is of the form $Y_{0}(t):=X_{0}\left(N_{0}(t)\right), t \in[0, b]$. 


\section{Convergence of stochastic integrals With Respect to martingales}

As in Section 2, let $\left(\Omega^{n}, \mathcal{F}^{n},\left(\mathcal{F}_{t}^{n}\right)_{t \in R_{+}}, \mathrm{P}^{n}\right)$ be a stochastic basis for every $n$, and let $\left\{M_{n}(t), \mathcal{F}_{t}^{n}, t \in \mathbf{R}_{+}, n \geq 0\right\}$ be a sequence of square integrable martingales whose trajectories do not have discontinuities of the second kind and are right continuous. Let $\mu_{n}(t):=\left\langle M_{n}\right\rangle(t)$ be the square characteristics of the above martingales. We consider the modifications for which the trajectories do not have discontinuities of the second kind and are right continuous. Also let $\left\{\xi_{n}(t), \mathcal{F}_{t}^{n}, t \in \mathbf{R}_{+}, n \geq 0\right\}$ be a sequence of $\mathcal{F}^{n}$-predictable processes.

Theorem 3. Assume that

$\left(\mathrm{A}_{5}\right)$ 1) $\left(\xi_{n}(t), M_{n}(t), \mu_{n}(t), t \in T_{b}\right) \Rightarrow\left(\xi_{0}(t), M_{0}(t), \mu_{0}(t), t \in T_{b}\right)$, for all $b>0$;

2) for all $t \in \mathbf{R}_{+}$,

$$
\sup _{n \geq 0} \mathrm{E}^{n} \int_{0}^{t} \xi_{n}^{2}(s) d \mu_{n}(s)<\infty
$$

3) for all $b>0$,

$$
\lim _{c \rightarrow \infty} \limsup _{n \rightarrow \infty} \mathrm{P}^{n}\left\{\sup _{0 \leq t \leq b}\left|\xi_{n}(t)\right| \geq c\right\}=0
$$

4) for all $b>0$,

$$
\lim _{k \rightarrow \infty} \limsup _{n \rightarrow \infty} \mathrm{E}^{n} \sum_{i=1}^{k_{b}+1} \omega_{i k} \xi_{n} \omega_{i k} \mu_{n}=0 ;
$$

5) $\lim _{\delta \rightarrow 0} \lim \sup _{n \rightarrow \infty} \sup _{\sigma \in \mathcal{T}_{b}\left(\mathcal{F}^{n}\right)} \mathrm{E}^{n}\left(\mu_{n}(\sigma+\delta)-\mu_{n}(\sigma)\right)=0$.

Then, for all $b>0$, the sequence of stochastic integrals weakly converges:

$$
\int_{0}^{\cdot} \xi_{n}(t) d M_{n}(t) \stackrel{D[0, b]}{\rightarrow} \int_{0}^{\cdot} \xi_{0} d M_{0}(t), \quad n \rightarrow \infty .
$$

Moreover the sequence of their square characteristics also weakly converges:

$$
\int_{0}^{\cdot} \xi_{n}^{2}(t) d \mu_{n}(t) \stackrel{D[0, b]}{\rightarrow} \int_{0}^{\cdot} \xi_{0}^{2} d \mu_{0}(t), \quad n \rightarrow \infty .
$$

Proof. Conditions $\left(\mathrm{A}_{5}\right), 1$ ) and 4) together with Theorem 1 imply the convergence of finite-dimensional distributions:

$$
\left(\int_{0}^{t} \xi_{n}^{2}(u) d \mu_{n}(u), t \in T_{b}\right) \Rightarrow\left(\int_{0}^{t} \xi_{0}^{2}(u) d \mu_{0}(u), t \in T_{b}\right), \quad n \rightarrow \infty .
$$

Further, $\left.\left(A_{5}\right), 1\right)$ implies that

$$
\sum_{i=1}^{k} \xi_{n}\left(t_{i-1 k}\right) \triangle_{i k} M_{n} \Rightarrow \sum_{i=1}^{k} \xi_{0}\left(t_{i-1 k}\right) \triangle_{i k} M_{0}, \quad n \rightarrow \infty .
$$

In its turn, $\left.\left(\mathrm{A}_{5}\right), 2\right)$ implies that, for all $n \geq 0$,

$$
\sum_{i=1}^{k} \xi_{n}\left(t_{i-1 k}\right) \Delta_{i k} M_{n} \rightarrow \int_{0}^{b} \xi_{n}(s) d M_{n}(s), \quad k \rightarrow \infty
$$


in probability. Further, for all $\alpha>0$,

$$
\begin{aligned}
\lim _{k \rightarrow \infty} \limsup _{n \rightarrow \infty} \mathrm{P}^{n}\left\{\left|\sum_{i=1}^{k} \xi_{n}\left(t_{i-1 k}\right) \Delta_{i k} M_{n}-\int_{0}^{b} \xi_{n}(s) d M_{n}(s)\right|>\alpha\right\} \\
=\lim _{k \rightarrow \infty} \limsup _{n \rightarrow \infty} \mathrm{P}^{n}\left\{\left|\int_{0}^{b} \varphi_{n}(s) d M_{n}(s)\right|>\alpha\right\} \\
\leq \lim _{C \rightarrow \infty} \limsup _{n \rightarrow \infty} \mathrm{P}^{n}\left\{\sup _{0 \leq t \leq b}\left|\xi_{n}(t)\right| \geq C\right\} \\
\quad+\alpha^{-2} \lim _{C \rightarrow \infty} \lim _{k \rightarrow \infty} \limsup _{n \rightarrow \infty} \mathrm{E}^{n} \int_{0}^{b}\left(\varphi_{n}^{C}(s)\right)^{2} d \mu_{n}(s) \\
\leq \alpha^{-2} \lim _{C \rightarrow \infty}\left(C \lim _{k \rightarrow \infty} \limsup _{n \rightarrow \infty} \mathrm{E}^{n} \sum_{i=1}^{k_{b}+1} \omega_{i k} \xi_{n} \omega_{i k} \mu_{n}\right)=0,
\end{aligned}
$$

where $\varphi_{n}(s):=\xi_{n}(s)-\xi_{n}\left(t_{i-1 k}\right)$ and $\varphi_{n}^{C}(s):=\xi_{n}(s) \wedge C-\xi_{n}\left(t_{i-1 k}\right) \wedge C, s \in \triangle_{i k}$.

Relatons (7)-(9) and Theorem 4.2 in [3] yield the weak convergence of the finitedimensional distributions:

$$
\int_{0}^{b} \xi_{n}(t) d M_{n}(t) \Rightarrow \int_{0}^{b} \xi_{0} d M_{0}(t), \quad n \rightarrow \infty .
$$

Moreover, for all $\delta>0$, condition $\left.\left(\mathrm{A}_{5}\right), 2\right)$ implies that

$$
\begin{aligned}
& \lim _{C \rightarrow \infty} \limsup _{n \rightarrow \infty} \mathrm{P}^{n}\left(\sup _{t \leq \delta}\left|\int_{0}^{t} \xi_{n}(u) d M_{n}(u)\right| \geq C\right) \\
& \leq \lim _{C \rightarrow \infty} \frac{1}{C^{2}} \limsup _{n \rightarrow \infty} \mathrm{E}^{n}\left(\sup _{t \leq \delta}\left|\int_{0}^{t} \xi_{n}(u) d M_{n}(u)\right|^{2}\right) \\
& \leq \lim _{C \rightarrow \infty} \limsup _{n \rightarrow \infty} \frac{C_{1}}{C^{2}} \mathrm{E}^{n} \int_{0}^{\delta} \xi_{n}^{2}(u) d \mu_{n}(u)=0 .
\end{aligned}
$$

Now we consider the following stochastic process:

$$
Z_{n}^{\sigma}(t, C)=\int_{0}^{t+\sigma} \xi_{n}^{C}(u) d M_{n}(u)-\int_{0}^{\sigma} \xi_{n}^{C}(u) d M_{n}(u),
$$

where $t \geq 0, \sigma \in \mathcal{T}_{b}\left(\mathcal{F}^{n}\right)$, and $\xi_{n}^{C}(u)=\xi_{n}(u) \wedge C$. This process is a martingale with respect to the flow of $\sigma$-algebras $\mathcal{F}_{t+\sigma}^{n}, t \in \mathbf{R}_{+}$. Then $\left.\left(\mathrm{A}_{5}\right), 3\right)$ and 5) together with Burkholder's inequality imply that

$$
\begin{aligned}
& \lim _{\delta \rightarrow 0} \limsup _{n \rightarrow \infty} \sup _{\sigma \in \mathcal{T}_{b}\left(\mathcal{F}^{n}\right)} \mathrm{P}^{n}\left(\sup _{t \leq \delta}\left|\int_{0}^{t+\sigma} \xi_{n}(u) d M_{n}(u)-\int_{0}^{\sigma} \xi_{n}(u) d M_{n}(u)\right| \geq \eta\right) \\
& \leq \lim _{C \rightarrow \infty} \limsup _{n \rightarrow \infty} \mathrm{P}^{n}\left(\left|\xi_{n}(t)\right| \geq C\right)+\frac{C_{2}}{\eta^{2}} \lim _{\delta \rightarrow 0} \limsup _{n \rightarrow \infty} \sup _{\sigma \in \mathcal{T}_{b}\left(\mathcal{F}^{n}\right)} \mathrm{E}^{n}\left[Z_{n}^{\sigma}(\delta, c)\right]^{2} \\
& \quad=\frac{C_{2}}{\eta^{2}} \lim _{\delta \rightarrow 0} \limsup _{n \rightarrow \infty} \sup _{\sigma \in \mathcal{T}_{b}\left(\mathcal{F}^{n}\right)} \mathrm{E}^{n}\left(\mu_{n}(\sigma+\delta)-\mu_{n}(\sigma)\right)=0 .
\end{aligned}
$$

Similarly to (12) one can prove that

$$
\lim _{C \rightarrow \infty} \limsup _{n \rightarrow \infty} \mathrm{P}^{n}\left(\sup _{t \leq \delta}\left|\int_{0}^{t} \xi_{n}^{2}(u) d \mu_{n}(u)\right| \geq C\right)=0
$$


and

$$
\begin{gathered}
\lim _{\delta \rightarrow 0} \limsup _{n \rightarrow \infty} \sup _{\sigma \in \mathcal{T}_{b}\left(\mathcal{F}^{n}\right)} \mathrm{P}^{n}\left(\sup _{t \leq \delta}\left|\int_{0}^{t+\sigma} \xi_{n}^{2}(u) d \mu_{n}(u)-\int_{0}^{\sigma} \xi_{n}^{2}(u) d \mu_{n}(u)\right| \geq \eta\right)=0, \\
\eta>0 .
\end{gathered}
$$

Now we obtain from (11)-(10) and Theorem 1 of [1, Part 2, Chapter 6 , $\S 3$, that the sequences $\int_{0}^{*} \xi_{n}(t) d M_{n}(t)$ and $\int_{0}^{*} \xi_{n}^{2}(t) d \mu_{n}(t)$ are relatively compact in the Skorokhod topology. The weak convergence of the finite-dimensional distributions (see relations (7) and (14)) imply the convergence of these sequences in the Skorokhod topology, as well.

Remark 2. One can assume that

$$
\mathrm{E}^{n} \int_{0}^{t} \xi_{n}^{2}(s) d \mu_{n}(s)<\infty, \quad t \in \mathbf{R}_{+}, \quad \lim _{C \rightarrow \infty} \limsup _{n \rightarrow \infty} \mathrm{P}^{n}\left(\mu_{n}(t) \geq C\right)=0, \quad t>0,
$$

instead of condition $\left.\left(A_{5}\right), 2\right)$.

Remark 3. As mentioned above, the conditions for the convergence of stochastic integrals with respect to martingales are studied in [2]. The main differences between conditions of the paper [2] and those of Theorem 2 are listed below.

1) It is not assumed in Theorem 3 that the martingales $M_{n}$ are continuous, while this is one of the assumption in the corresponding theorem in [2].

2) The counterpart of assumption $\left.\left(A_{5}\right), 4\right)$ in 2 is

$$
\lim _{k \rightarrow \infty} \limsup _{n \rightarrow \infty} \mathrm{E}^{n} \sum_{i=1}^{k_{b}+1} \int_{t_{i-1 k}}^{t_{i k}} \triangle_{i k} \xi_{n}(t) d \mu_{n}(t)=0
$$

for all $b>0$.

\section{Convergence of Stochastic integrals With Respect to semimartingales IN TERMS OF THE CANONICAL DECOMPOSITION}

Now let $\left(\Omega^{n}, \mathcal{F}^{n},\left(\mathcal{F}_{t}^{n}\right)_{t \in \mathbf{R}_{+}}, \mathrm{P}^{n}\right)$ be a stochastic basis for every $n$, and let

$$
\left\{X_{n}(t), \mathcal{F}_{t}^{n}, t \in \mathbf{R}_{+}, n \in \mathbf{Z}_{+}\right\}
$$

be a sequence of semimartingales that admits the following decomposition:

$$
X_{n}(t)=X_{n}^{0}+M_{n}(t)+B_{n}(t)
$$

where $\left\{M_{n}(t), \mathcal{F}_{t}^{n}, t \in \mathbf{R}_{+}, n \in \mathbf{Z}_{+}\right\}$is a sequence of square integrable martingales whose trajectories do not have discontinuities of the second kind and are right continuous, and $\left\{B_{n}(t), t \in \mathbf{R}_{+}, n \in \mathbf{Z}_{+}\right\}$is a sequence of processes of bounded variation whose trajectories do not have discontinuities of the second kind and are right continuous. We denote by $\Delta Y(t)=Y(t)-Y(t-0)$ the jump of a process $Y$ with such trajectories at a point $t$.

Let $\mu_{n}(t):=\left\langle M_{n}\right\rangle(t)$ be square characteristics of the corresponding martingales. We also consider a sequence $\left\{\xi_{n}(t), \mathcal{F}_{t}^{n}, t \in \mathbf{R}_{+}, n \geq 0\right\}$ of $\mathcal{F}^{n}$-predictable processes satisfying the following conditions:

$$
\mathrm{E}^{n} \int_{0}^{b} \xi_{n}^{2}(t) d \mu_{n}(t)<\infty, \quad\left|\int_{0}^{b} \xi_{n}(t) d B_{n}(t)\right|<\infty \quad \mathrm{P}^{n} \text {-a.s., } \quad b \in \mathbf{R}_{+}, n \in \mathbf{Z}_{+},
$$

where the integral $\int_{0}^{b} \xi_{n}(t) d B_{n}(t)$ is understood in the Riemann-Stieltjes sense.

Define

$$
\int_{0}^{t} \xi_{n}(s) d X_{n}(s):=\int_{0}^{t} \xi_{n}(s) d M_{n}(s)+\int_{0}^{t} \xi_{n}(s) d B_{n}(s) .
$$


Note that the definition of the integral on the left hand side is correct, since the right hand side is invariant with respect to all such decompositions.

In what follows we need the notion of density of a sequence of stochastic processes which coincides with the notion of density of a sequence of the corresponding probability measures. We also need the definition of the $C$-density.

Definition 1. A sequence of processes $X_{n}$ is called $C$-dense if the sequence is dense and every limit point of the sequence of the corresponding distributions is the distribution of a continuous process.

Theorem 4. Let $\left\{X_{n}(t), \mathcal{F}_{t}^{n}, t \in \mathbf{R}_{+}, n \in \mathbf{Z}_{+}\right\}$be a sequence of semimartingales that admits decomposition (15). Assume that

1) for all $b>0$,

$$
\left(\xi_{n}(t), M_{n}(t), B_{n}(t), \mu_{n}(t), t \in T_{b}\right) \Rightarrow\left(\xi_{0}(t), M_{0}(t), B_{0}(t), \mu_{0}(t), t \in T_{b}\right) ;
$$

2) for all $t \in \mathbf{R}_{+}$,

3) for all $b>0$,

$$
\sup _{n \geq 0} \mathrm{E}^{n} \int_{0}^{t} \xi_{n}^{2}(s) d \mu_{n}(s)<\infty
$$

$$
\lim _{C \rightarrow \infty} \limsup _{n \rightarrow \infty} \mathrm{P}^{n}\left\{\sup _{0 \leq t \leq b}\left|\xi_{n}(t)\right| \geq C\right\}=0
$$

4) for all $b>0$,

$$
\lim _{k \rightarrow \infty} \limsup _{n \rightarrow \infty} \mathrm{E}^{n} \sum_{i=1}^{k_{b}+1} \omega_{i k} \xi_{n} \omega_{i k} B_{n}=0 ;
$$

5) for all $\alpha>0$ and $b>0$,

$$
\lim _{\delta \rightarrow 0} \limsup _{n \rightarrow \infty} \mathrm{P}^{n}\left\{\triangle_{D}\left(\left|B_{n}\right|(\cdot), \delta, b\right)>\alpha\right\}=0 ;
$$

6) for all $b>0$,

$$
\lim _{k \rightarrow \infty} \limsup _{n \rightarrow \infty} \mathrm{E}^{n} \sum_{i=1}^{k_{b}+1} \omega_{i k} \xi_{n} \omega_{i k} \mu_{n}=0 ;
$$

7) $\lim _{\delta \rightarrow 0} \lim \sup _{n \rightarrow \infty} \sup _{\sigma \in \mathcal{T}_{b}\left(\mathcal{F}^{n}\right)} \mathrm{E}^{n}\left(\mu_{n}(\sigma+\delta)-\mu_{n}(\sigma)\right)=0$;

8) for all $\alpha>0$ and $b>0$,

$$
\limsup _{n \rightarrow \infty} \mathrm{P}^{n}\left\{\sup _{t \in[0, b]}\left|\Delta B_{n}(t)\right|>\alpha\right\}=0 .
$$

Then the family of stochastic integrals $\int_{0}^{\cdot} \xi_{n}(t) d X_{n}(t)$ weakly converges, namely

$$
\int_{0}^{\cdot} \xi_{n}(t) d X_{n}(t) \stackrel{D[0, b]}{\rightarrow} \int_{0}^{\cdot} \xi_{0} d X_{0}(t), \quad n \rightarrow \infty .
$$

Proof. First we prove the convergence of the finite-dimensional distributions similarly to the proofs of Theorems 1 and 2, Condition 1) implies that

$$
\begin{aligned}
\sum_{i=1}^{k} \xi_{n}\left(t_{i-1 k}\right) \triangle_{i k} M_{n}+\sum_{i=1}^{k} \xi_{n}\left(t_{i-1 k}\right) \triangle_{i k} B_{n} \\
\Rightarrow \sum_{i=1}^{k} \xi_{0}\left(t_{i-1 k}\right) \triangle_{i k} M_{0}+\sum_{i=1}^{k} \xi_{0}\left(t_{i-1 k}\right) \triangle_{i k} B_{0}, \quad n \rightarrow \infty
\end{aligned}
$$


Thus

$$
\begin{aligned}
\sum_{i=1}^{k} \xi_{n}\left(t_{i-1 k}\right) \Delta_{i k} M_{n}+\sum_{i=1}^{k} \xi_{n}\left(t_{i-1 k}\right) \Delta_{i k} B_{n} \\
\quad \rightarrow \int_{0}^{b} \xi_{n}(s) d M_{n}(s)+\int_{0}^{b} \xi_{n}(s) d B_{n}(s), \quad k \rightarrow \infty,
\end{aligned}
$$

in probability, where the convergence of integral sums with $M_{n}$ follows from 2), while the convergence of sums with $B_{n}$ follows from the definition of the Riemann-Stieltjes integral.

Further, relations (5) and (9) together with conditions and 4) and 6) imply that

$$
\begin{gathered}
\lim _{k \rightarrow \infty} \limsup _{n \rightarrow \infty} \mathrm{P}^{n}\left\{\mid \sum_{i=1}^{k} \xi_{n}\left(t_{i-1}\right) \Delta_{i k} M_{n}+\sum_{i=1}^{k} \xi_{n}\left(t_{i-1 k}\right) \Delta_{i k} B_{n}\right. \\
\left.\quad-\int_{0}^{b} \xi_{n}(s) d M_{n}(s)-\int_{0}^{b} \xi_{n}(s) d B_{n}(s) \mid>\alpha\right\} \\
\leq \lim _{k \rightarrow \infty} \limsup _{n \rightarrow \infty} \mathrm{P}^{n}\left\{\left|\sum_{i=1}^{k} \xi_{n}\left(t_{i-1 k}\right) \Delta_{i k} M_{n}-\int_{0}^{b} \xi_{n}(s) d M_{n}(s)\right|>\frac{\alpha}{2}\right\} \\
+\lim _{k \rightarrow \infty} \limsup _{n \rightarrow \infty} \mathrm{P}^{n}\left\{\left|\sum_{i=1}^{k} \xi_{n}\left(t_{i-1 k}\right) \Delta_{i k} B_{n}-\int_{0}^{b} \xi_{n}(s) d B_{n}(s)\right|>\frac{\alpha}{2}\right\}=0
\end{gathered}
$$

for all $\alpha>0$. The convergence of the finite-dimensional distributions

$$
\begin{aligned}
& \int_{0}^{t} \xi_{n}(s) d X_{n}(s)=\int_{0}^{t} \xi_{n}(s) d B_{n}(s)+\int_{0}^{t} \xi_{n}(s) d M_{n}(s) \\
& \quad \Rightarrow \int_{0}^{t} \xi_{0}(s) d B_{0}(s)+\int_{0}^{t} \xi_{0}(s) d M_{0}(s)=\int_{0}^{t} \xi_{0}(s) d X_{0}(s), \quad n \rightarrow \infty,
\end{aligned}
$$

follows from (16)-(18) and Theorem 4.2 in 3 .

Conditions 5) and 7) imply that the sequences $\int_{0}^{t} \xi_{n}(s) d B_{n}(s)$ and $\int_{0}^{t} \xi_{n}(s) d M_{n}(s)$ are dense (this is proved in Theorems 1 and 2, respectively).

Conditions 3) and 8) yield

$$
\limsup _{n \rightarrow \infty} \mathrm{P}^{n}\left\{\sup _{t \in[0, b]}\left|\Delta \int_{0}^{t} \xi_{n}(s) d B_{n}(s)\right|>\alpha\right\}=0
$$

for all $\alpha>0$ and $b>0$.

Since $\int_{0}^{t} \xi_{n}(s) d B_{n}(s)$ is dense, relation (20) implies that the sequence of integrals is $C$-dense (see Theorem 3.26 in [5]).

The sequence $\int_{0}^{t} \xi_{n}(s) d B_{n}(s)$ is $C$-dense, the sequence $\int_{0}^{t} \xi_{n}(s) d M_{n}(s)$ is dense, and thus the sum of the corresponding sequences

$$
\int_{0}^{t} \xi_{n}(s) d X_{n}(s)
$$

is also dense (see Theorem 3.33 in [5]), and the proof is complete.

We generalize Theorem 4 to the multidimensional case. Let

$$
\left\{X_{n}(t), \mathcal{F}_{t}^{n}, t \in \mathbf{R}_{+}\right\}=\left\{\left(X_{n}^{1}(t), X_{n}^{2}(t), \ldots, X_{n}^{d}(t)\right), \mathcal{F}_{t}^{n}, t \in \mathbf{R}_{+}, n \in \mathbf{Z}_{+}\right\}
$$


be a sequence of $d$-dimensional semimartingales whose components admit the following decomposition:

$$
X_{n}^{j}(t)=X_{n}^{j}(0)+M_{n}^{j}(t)+B_{n}^{j}(t), \quad 1 \leq j \leq d,
$$

where $\left\{M_{n}^{j}(t), \mathcal{F}_{t}^{n}, t \in \mathbf{R}_{+}, n \in \mathbf{Z}_{+}\right\}$is a sequence of square integrable martingales whose trajectories are right continuous and do not have discontinuities of the second kind and where $\left\{B_{n}^{j}(t), t \in \mathbf{R}_{+}, n \in \mathbf{Z}_{+}\right\}$is a sequence of processes of bounded variation whose trajectories do not have discontinuities of the second kind and are right continuous. Let $\mu_{n}^{j}(t):=\left\langle M_{n}^{j}\right\rangle(t)$ be square characteristics of the corresponding martingales. Also let

$$
\left\{\xi_{n}^{j}(t), \mathcal{F}_{t}^{n}, t \in \mathbf{R}_{+}, n \in \mathbf{Z}_{+}, 1 \leq j \leq d\right\}
$$

be a sequence of $\mathcal{F}^{n}$-predictable processes satisfying the following conditions:

$$
\mathrm{E}^{n} \int_{0}^{b}\left(\xi_{n}^{j}\right)^{2}(t) d \mu_{n}^{j}(t)<\infty, \quad \int_{0}^{b} \xi_{n}^{j}(t) d B_{n}^{j}(t)<\infty
$$

$P^{n}$-almost surely for $b \in \mathbf{R}_{+}, n \in \mathbf{Z}_{+}$, and $1 \leq j \leq d$.

Put

$$
\int_{0}^{t}\left(\xi_{n}(t), d X_{n}(t)\right):=\sum_{j=1}^{d} \int_{0}^{t} \xi_{n}^{j}(t) d X_{n}^{j}(t) .
$$

Theorem 5. Let $\left\{X_{n}(t), \mathcal{F}_{t}^{n}, t \in \mathbf{R}_{+}, n \in \mathbf{Z}_{+}\right\}$be a sequence of semimartingales admitting decomposition (21). Assume that

1) for all $b>0$ and for all $1 \leq j \leq d$,

$$
\left(\xi_{n}(t), M_{n}(t), B_{n}(t), \mu_{n}(t), t \in T_{b}\right) \Rightarrow\left(\xi_{0}(t), M_{0}(t), B_{n}(0), \mu_{0}(t), t \in T_{b}\right) ;
$$

2) for all $t \in \mathbf{R}_{+}$,

$$
\sup _{n \geq 0} \mathrm{E}^{n} \int_{0}^{t}\left(\xi_{n}^{j}\right)^{2}(s) d \mu_{n}^{j}(s)<\infty
$$

3) for all $b>0$,

$$
\lim _{c \rightarrow \infty} \limsup _{n \rightarrow \infty} \mathrm{P}^{n}\left\{\sup _{0 \leq t \leq b}\left|\xi_{n}^{j}(t)\right| \geq c\right\}=0
$$

4) for all $b>0$,

$$
\lim _{k \rightarrow \infty} \limsup _{n \rightarrow \infty} \mathrm{E}^{n} \sum_{i=1}^{k_{b}+1} \omega_{i k} \xi_{n}^{j} \omega_{i k} B_{n}^{j}=0 ;
$$

5) for all $\alpha>0$ and $b>0$,

$$
\lim _{\delta \rightarrow 0} \limsup _{n \rightarrow \infty} \mathrm{P}^{n}\left\{\triangle_{D}\left(\left|B_{n}^{j}\right|(\cdot), \delta, b\right)>\alpha\right\}=0 ;
$$

6) for all $b>0$,

$$
\lim _{k \rightarrow \infty} \limsup _{n \rightarrow \infty} \mathrm{E}^{n} \sum_{i=1}^{k_{b}+1} \omega_{i k} \xi_{n}^{j} \omega_{i k} \mu_{n}^{j}=0 ;
$$

7) $\lim _{\delta \rightarrow 0} \lim \sup _{n \rightarrow \infty} \sup _{\sigma \in \mathcal{T}_{b}\left(\mathcal{F}^{n}\right)} \mathrm{E}^{n}\left(\mu_{n}^{j}(\sigma+\delta)-\mu_{n}^{j}(\sigma)\right)=0$;

8) for all $\alpha>0$ and $b>0$,

$$
\limsup _{n \rightarrow \infty} \mathrm{P}^{n}\left\{\sup _{t \in[0, b]}\left|\Delta B_{n}^{j}(t)\right|>\alpha\right\}=0 .
$$


Then the family of stochastic integrals $\int_{0}^{*}\left(\xi_{n}(s), d X_{n}(s)\right)$ weakly converges:

$$
\int_{0}^{\cdot}\left(\xi_{n}(s), d X_{n}(s)\right) \stackrel{D[0, b]}{\rightarrow} \int_{0}^{\cdot}\left(\xi_{0}(s), d X_{0}(s)\right), \quad n \rightarrow \infty .
$$

Proof. The proof follows the lines of the proof of Theorem 4. Condition 1) implies that

$$
\begin{aligned}
& \sum_{j=1}^{d} \sum_{i=1}^{k} \xi_{n}^{j}\left(t_{i-1 k}\right) \triangle_{i k} M_{n}^{j}+\sum_{j=1}^{d} \sum_{i=1}^{k} \xi_{n}^{j}\left(t_{i-1 k}\right) \triangle_{i k} B_{n}^{j} \\
& \Rightarrow \sum_{j=1}^{d} \sum_{i=1}^{k} \xi_{0}^{j}\left(t_{i-1 k}\right) \triangle_{i k} M_{0}^{j}+\sum_{j=1}^{d} \sum_{i=1}^{k} \xi_{0}^{j}\left(t_{i-1 k}\right) \triangle_{i k} B_{0}^{j}, \quad n \rightarrow \infty .
\end{aligned}
$$

Condition 2) yields, in particular, that

$$
\begin{aligned}
& \sum_{j=1}^{d} \sum_{i=1}^{k} \xi_{n}^{j}\left(t_{i-1 k}\right) \Delta_{i k} M_{n}^{j}+\sum_{j=1}^{d} \sum_{i=1}^{k} \xi_{n}^{j}\left(t_{i-1 k}\right) \Delta_{i k} B_{n}^{j} \\
& \rightarrow \int_{0}^{b} \sum_{j=1}^{d} \xi_{n}^{j}(s) d M_{n}^{j}(s)+\sum_{j=1}^{d} \int_{0}^{b} \xi_{n}^{j}(s) d B_{n}^{j}(s), \quad k \rightarrow \infty,
\end{aligned}
$$

in probability for all $n \geq 0$.

Further, similarly to (18) we derive from 4) and 6) that

$$
\begin{aligned}
& \operatorname{lims}_{k \rightarrow \infty} \limsup _{n \rightarrow \infty} \mathrm{P}^{n}\left\{\mid \sum_{j=1}^{d} \sum_{i=1}^{k} \xi_{n}^{j}\left(t_{i-1 k}\right) \Delta_{i k} M_{n}^{j}+\sum_{j=1}^{d} \sum_{i=1}^{k} \xi_{n}^{j}\left(t_{i-1 k}\right) \Delta_{i k} B_{n}^{j}\right. \\
& \left.\quad-\sum_{j=1}^{d} \int_{0}^{b} \xi_{n}^{j}(s) d M_{n}^{j}(s)-\sum_{j=1}^{d} \int_{0}^{b} \xi_{n}^{j}(s) d B_{n}^{j}(s) \mid>\alpha\right\} \\
& \leq \sum_{j=1}^{d}\left(\lim _{k \rightarrow \infty} \limsup _{n \rightarrow \infty} \mathrm{P}^{n}\left\{\left|\sum_{i=1}^{k} \xi_{n}^{j}\left(t_{i-1 k}\right) \Delta_{i k} M_{n}^{j}-\int_{0}^{b} \xi_{n}^{j}(s) d M_{n}^{j}(s)\right|>\frac{\alpha}{2 d}\right\}\right. \\
& \left.\quad+\lim _{k \rightarrow \infty} \limsup _{n \rightarrow \infty} \mathrm{P}^{n}\left\{\left|\sum_{i=1}^{k} \xi_{n}^{j}\left(t_{i-1 k}\right) \Delta_{i k} B_{n}^{j}-\int_{0}^{b} \xi_{n}^{j}(s) d B_{n}^{j}(s)\right|>\frac{\alpha}{2 d}\right\}\right) \\
& \quad \text { for all } \alpha>0 .
\end{aligned}
$$

Relations (22)-(24) and Theorem 4.2 of 3 imply the weak convergence of the finitedimensional distributions:

$$
\begin{gathered}
\int_{0}^{t}\left(\xi_{0}(s), d X_{0}(s)\right)=\int_{0}^{t}\left(\xi_{n}(s) d B_{n}(s)\right)+\int_{0}^{t}\left(\xi_{n}(s) d M_{n}(s)\right) \\
=\sum_{j=1}^{d} \int_{0}^{t} \xi_{n}^{j}(s) d B_{n}^{j}(s)+\sum_{j=1}^{d} \int_{0}^{t} \xi_{n}^{j}(s) d M_{n}^{j}(s) \\
\Rightarrow \sum_{j=1}^{d} \int_{0}^{t} \xi_{0}^{j}(s) d B_{0}^{j}(s)+\sum_{j=1}^{d} \int_{0}^{t} \xi_{0}^{j}(s) d M_{0}^{j}(s) \\
=\int_{0}^{t}\left(\xi_{0}(s), d B_{0}(s)\right)+\int_{0}^{t}\left(\xi_{0}(s), d M_{0}(s)\right)=\int_{0}^{t}\left(\xi_{0}(s), d X_{0}(s)\right), \\
n \rightarrow \infty .
\end{gathered}
$$


It follows from conditions 5) and 7) that the sequences

$$
\int_{0}^{t} \xi_{n}^{j}(s) d B_{n}^{j}(s) \quad \text { and } \quad \int_{0}^{t} \xi_{n}^{j}(s) d M_{n}^{j}(s)
$$

are dense for all $1 \leq j \leq d$.

Similarly to the proof of the preceding theorem, the above reasoning and conditions 7) and 8) imply that the sequences $\int_{0}^{t} \xi_{n}^{j}(s) d B_{n}^{j}(s)$ and $\int_{0}^{t} \xi_{n}^{j}(s) d X_{n}^{j}(s)$ are $C$-dense for all $1 \leq j \leq d$ (see Theorem 3.26 in [5]).

Furthermore, the sequence $\int_{0}^{t} \xi_{n}(s) d X_{n}(s)$ is $C$-dense (thus this sequence is dense) in view of Theorem 3.33 of [5]. This completes the proof of the theorem.

\section{Conditions For the STABility of Integrands \\ IN THE MULTIDIMENSIONAL CASE}

In this section, we exhibit the conditions on integrators and integrands for the multidimensional case that guarantee the weak convergence if the integrals converge. In other words, we solve an "inverse" problem for the weak convergence. This problem occurs in financial mathematics when one needs to investigate the limit behavior of a certain class of strategies if the capitals converge. This problem for the one-dimensional case is solved in [6]. In this section, we study the multidimensional case. Some applications of these results will be presented in the second part of the paper.

Let $(\Omega, \mathcal{F}, \mathrm{P})$ be a complete probability space.

Let a process $X(t)$ be a square integrable semimartingale that admits the following decomposition:

$$
X^{j}(t)=B^{j}(t)+M^{j}(t),
$$

where $M^{j}$ is a square integrable martingale for all $1 \leq j \leq d$ with square characteristics $\mu^{j}$, and where $B^{j}$ is a predictable process of integrable variation. Assume that the filtration $\left\{\mathcal{F}_{t}, t \in[0, T]\right\}$ is generated by the process $X$.

For the integrands we consider $\mathcal{F}_{t}$-predictable processes

$$
\xi(t)=\left(\xi^{1}(t), \ldots, \xi^{d}(t)\right) \in \mathbf{R}^{d}
$$

such that

$$
\mathrm{E} \int_{0}^{T}\left(\xi^{j}(s)\right)^{2} d \mu^{j}(s)<\infty, \quad \mathrm{E}\left(\int_{0}^{T}\left|\xi^{j}(s)\right| d\left|B^{j}\right|(s)\right)^{2}<\infty, \quad 1 \leq j \leq d .
$$

We assume that the processes $X^{j}$ are linearly independent. This means that if $\zeta \in \mathbb{R}^{d}$ is $\mathcal{F}_{t}$-predictable and

$$
\sum_{j=1}^{d} \int_{0}^{t} \zeta^{j}(s) d X^{j}(s)=0
$$

almost surely for $t \in[0, T]$, then $\left(\zeta^{1}(t), \ldots, \zeta^{d}(t)\right)=0$ almost surely for all $t \in[0, T]$.

As before, we put $\int_{0}^{t}(\xi(s), d X(s)):=\sum_{j=1}^{d} \int_{0}^{t} \xi^{j}(s) d X^{j}(s)$.

Lemma 1. Let stochastic processes $(\xi(t), X(t))$ satisfy the above conditions. Assume that

$$
\left(\xi(t), X(t), \int_{0}^{t}(\xi(s), d X(s)), t \in[0, T]\right) \stackrel{d}{=}(\zeta(t), Y(t), Z(t), t \in[0, T])
$$

for some processes $\zeta, Y$, and $Z$.

Then $Y$ is a semimartingale and $Z(t)=\int_{0}^{t}(\zeta(s), d Y(s))$ for all $t \in[0, T]$. 
Proof. Note that if $S$ is an $\mathcal{F}_{t}$-predictable process such that, for all sequences $\left\{H_{n}\right.$, $n \geq 1$ \} of simple $\mathcal{F}_{t}$-predictable processes converging to $H$ in probability, the sequences $\int_{0}^{T} H_{n}(t) d S(t)$ converge to $\int_{0}^{T} H(t) d S(t)$ (see 9 ), then $S$ is a semimartingale with respect to $\left\{\mathcal{F}_{t}, t \in[0, T]\right\}$. Thus the semimartingale property with respect to the natural filtration depends only on the distribution of the process. Thus $Y$ is a semimartingale.

Further, if the processes $\xi$ and $X$ satisfy the above conditions, then the integral $\int_{0}^{t}(\xi(s), d X(s))$ is defined as the limit in probability of the integral sums

$$
S(\pi, \xi, X)=\sum_{k=1}^{n}\left(\xi\left(t_{k-1}\right), X\left(t_{k}\right)-X\left(t_{k-1}\right)\right)
$$

as the diameter of the partition $\pi=\left\{0=t_{0}<t_{1}<\cdots<t_{n}=t\right\}$ decreases.

The joint distribution of these sums and of $\int_{0}^{t} \xi(s) d X(s)$ is the same as that of the sums $S(\pi, \zeta, Y)$ and $Z(t)$. Thus $S(\pi, \zeta, Y) \stackrel{\mathrm{P}}{\rightarrow} Z(t),|\pi| \rightarrow 0$, whence

$$
Z(t)=\int_{0}^{t}(\zeta(s), d Y(s))
$$

Let $\left\{\xi_{n}(t), n \in \mathbf{Z}_{+}\right\}=\left\{\left(\xi_{n}^{1}(t), \ldots, \xi_{n}^{d}(t)\right), n \in \mathbf{Z}_{+}\right\}$be a sequence of predictable $d$ dimensional processes satisfying condition (27).

Theorem 6. Let $X$ and $\xi_{n}$ satisfy the above conditions. Assume also that

1) for all $0 \leq t \leq T$,

$$
\int_{0}^{t}\left(\xi_{n}(s), d X(s)\right) \stackrel{\mathrm{P}}{\rightarrow} \int_{0}^{t}\left(\xi_{0}(s), d X(s)\right) \quad \text { as } n \rightarrow \infty ;
$$

2) the sequence of measures corresponding to the processes $\left\{\xi_{n}(\cdot), n \in \mathbf{Z}_{+}\right\}$is weakly precompact in the space $D[0, T]$.

Then $\xi_{n}(\cdot) \rightarrow \xi_{0}(\cdot), n \rightarrow \infty$, in $D[0, T]$.

Proof. We prove that every sequence $\left\{\xi_{n}, n \geq 0\right\}$ contains a subsequence that weakly converges to $\xi_{0}$. Let $\left\{\xi_{n_{k}}, k \geq 0\right\}$ be a subsequence of $\left\{\xi_{n}, n \geq 0\right\}$. The weak precompactness implies that a certain subsequence of $\left\{\xi_{n_{k}}, k \geq 0\right\}$ is weakly convergent. For simplicity, we assume that the sequence $\left\{\xi_{n}, n \geq 0\right\}$ itself is weakly convergent in $D[0, T]$.

Moreover, we assume that the sequence of vectors $\left(\xi_{0}(\cdot), \int_{0}^{\cdot}\left(\xi_{0}(s), d X(s)\right), \xi_{n}(\cdot), X(\cdot)\right)$ weakly converges (recall that the whole sequence is precompact, since $\left\{\xi_{n}, n \geq 0\right\}$ is weakly precompact). Denote its limit by

$$
\left(\zeta_{0}(\cdot), Z(\cdot), \zeta(\cdot), Y(\cdot)\right) \text {. }
$$

We obtain from Lemma 1 that $Z(t)=\int_{0}^{t}\left(\zeta_{0}(s), d Y(s)\right)$.

Now we show that $\xi_{0} \stackrel{d}{=} \zeta$.

According to [7, Theorem 2.2] we obtain the weak convergence in $D[0, T]$ :

$$
\begin{aligned}
\left(\xi_{0}(\cdot),\right. & \left.\int_{0}^{\cdot}\left(\xi_{0}(s), d X(s)\right), \xi_{n}(\cdot), X(\cdot), \int_{0}^{\cdot} \xi_{n}(s) d X(s)\right) \\
\quad \Rightarrow & \left(\zeta_{0}(\cdot), \int_{0}^{\cdot}\left(\zeta_{0}(s), d Y(s)\right), \zeta(\cdot), Y(\cdot), \int_{0}^{\cdot}(\zeta(s), d Y(s))\right) .
\end{aligned}
$$

In fact, Theorem 2.2 of [7] states only that the three last components, namely the integrands, integrators, and integrals are jointly convergent. Nevertheless, we obtain the convergence of extended vectors, since if the additional components are attached to the integrands, while the integrator is appended with zeros, then the stochastic integral has the same value. 
The convergence in probability means that, for all $\varepsilon>0$,

$$
\mathrm{P}\left(\left|\int_{0}^{t}\left(\xi_{n}(s), d X(s)\right)-\int_{0}^{t}\left(\xi_{0}(s), d X(s)\right)\right|>\varepsilon\right) \rightarrow 0, \quad n \rightarrow \infty .
$$

On the other hand, the weak convergence (28) implies that

$$
\mathrm{P}\left(\left|\int_{0}^{t}(\zeta(s), d Y(s))-\int_{0}^{t}\left(\zeta_{0}(s), d Y(s)\right)\right|>\varepsilon\right)=0 .
$$

Therefore

$$
\int_{0}^{t}(\zeta(s), d Y(s))=\int_{0}^{t}\left(\zeta_{0}(s), d Y(s)\right)
$$

for all $t$.

The condition that the processes $X^{j}$ are "linearly independent" is expressed in terms of $\mathcal{F}_{t}$-predictable processes. On the other hand, the filtration $\left\{\mathcal{F}_{t}, t \in[0, t]\right\}$ is generated by the process $X$; thus, this condition remains true if we use another process $Y$ with the same distribution. In particular, the latter equality implies that $\zeta(t)=\zeta_{0}(t)$ almost surely for all $t \in[0, T]$. Hence $\xi_{0} \stackrel{d}{=} \zeta$ and the weak convergence $\xi_{n} \rightarrow \zeta, n \rightarrow \infty$, completes the proof of the theorem.

Remark 4. Theorem [6 may not hold if one assumes the weak convergence instead of the convergence in probability stated in condition 1). Indeed, if $d=1, X=B$ is a Brownian motion, and $\xi_{n} \equiv-1, \xi_{0} \equiv 1$, then

$$
\left\{\int_{0}^{t} \xi_{n}(t) d X(t), t \in[0, T]\right\} \stackrel{d}{=}\left\{\int_{0}^{t} \xi_{0}(t) d X(t), t \in[0, T]\right\}
$$

while $\xi_{n}$ does not converge to $\xi$ in any sense.

\section{Concluding Remarks}

Sufficient conditions for the weak convergence of stochastic integrals with respect to processes of bounded variation as well as with respect to martingales or semimartingales are studied in the paper. In particular, we proved the convergence of the corresponding probability measures in the Skorokhod space $D[0, b], b>0$, and for the multidimensional case.

The conditions on the integrators are imposed on components of an arbitrary fixed decomposition (not necessarily canonical decomposition), on the square integrable martingale, and on the process of bounded variation. An example of possible applications of the theorem on the convergence of stochastic integrals with respect to processes of bounded variation is presented in the case of risk processes. We also solved the "inverse" problem for the weak convergence that occurs in financial mathematics. This problem concerns the behavior of a certain class of strategies if the capitals converge. This application will be discussed in the second part of the paper in more detail.

\section{BIBLIOGRAPHY}

1. R. Sh. Liptser and A. N. Shiryaev, Theory of Maringales, Nauka, Moscow, 1986; English transl., Kluwer Academic Publishers Group, Dordrecht, 1989. MR1022664 (90j:60046)

2. Yu. S. Mishura, Some limit theorems for stochastic integrals with respect to a martingale and their applications, Teor. Veroyatnost. Mat. Statist. 22 (1980), 104-118; English transl. in Theory Probab. Math. Statist. 22 (1981), 115-129.

3. P. Billingsley, Convergence of Probability Measures, John Wiley \& Sons, 1968. MR0233396 $(38: 1718)$

4. J. Garrido, Weak convergence of risk processes, Insurance and Risk Theory, Nato Anvanced Study Institute on Insurance and Risk Theory, 1985, pp. 349-360. MR864549 (87j:60011) 
5. J. Jacod and A. N. Shiryaev, Limit Theorems for Stochastic Processes, Springer-Verlag, New York, 1987. MR959133 (89k:60044)

6. J. Jacod, S. Meleard, and P. Protter, Explicit form and robustness of martingale representations, Ann. Probab. 28 (2000), no. 4, 1747-1780. MR.1813842 (2001m:60127)

7. T. Kurtz and P. Protter, Weak limit theorems for stochastic integrals and stochastic differential equations, Ann. Probab. 19 (1991), no. 3, 1035-1070. MR.1112406 (92k:60130)

8. Yu. S. Mishura and D. S. Silvestrov, Limit theorems for stochastic Riemann-Stieltjes integrals, Theory Stoch. Process. 10 (26) (2004), no. 1-2, 122-140. MR2327856 (2008g:60169)

9. P. Protter, Stochastic Integration and Differential Equations, 2nd ed., Stochastic Modelling and Applied Probability, vol. 21, Springer, Berlin, 2005. MR.2273672 (2008e:60001)

Department of Probability Theory, Statistics, and Actuarial Mathematics, Faculty for Mechanics and Mathematics, National Taras Shevchenko University, Academician Glushkov Avenue 2, Kiev 03127, Ukraine

Department of Probability Theory, Statistics, and Actuarial Mathematics, Faculty for Mechanics and Mathematics, National Taras Shevchenko University, Academician Glushkov Avenue 2, Kiev 03127, Ukraine

E-mail address: zhora@univ.kiev.ua

Department of Probability Theory, Statistics, and Actuarial Mathematics, Faculty for Mechanics and Mathematics, National Taras Shevchenko University, Academician Glushkov Avenue 2, Kiev 03127, Ukraine

E-mail address: Yuhnovskiy@hq.eximb.com

Received 10/JUL/2009

Translated by N. SEMENOV 